\title{
Luxembourg SUMO Traffic (LuST) Scenario: 24 Hours of Mobility for Vehicular Networking Research
}

\author{
Lara CODECA, Raphael FRANK, Thomas ENGEL \\ Interdisciplinary Centre for Security, Reliability and Trust \\ University of Luxembourg, 2721, Luxembourg \\ Emails: lara.codeca@uni.lu raphael.frank@uni.lu thomas.engel@uni.lu
}

\begin{abstract}
Different research communities varying from telecommunication to traffic engineering are working on problems related to vehicular traffic congestion, intelligent transportation systems, and mobility patterns using information collected from a variety of sensors. To test the solutions, the first step is to use a vehicular traffic simulator with an appropriate scenario in order to reproduce realistic mobility patterns. Many mobility simulators are available, and the choice is usually done based on the size and type of simulation required, but a common problem is to find a realistic traffic scenario. In order to evaluate and compare new communication protocols for vehicular networks, it is necessary to use a wireless network simulator in combination with a vehicular traffic simulator. This additional step introduces further requirements for the scenario. The aim of this work is to provide a scenario able to meet all the common requirements in terms of size, realism and duration, in order to have a common basis for the evaluations. In the interest of building a realistic scenario, we decided to start from a real city with a standard topology common in mid-size European cities, and real information concerning traffic demands and mobility patterns. In this paper we show the process used to build the Luxembourg SUMO Traffic (LuST) Scenario, and present a summary of its characteristics together with an overview of its possible use cases.
\end{abstract}

Index Terms-Vehicle-to-X Simulation, Infrastructure-to-X Simulation, Scenario Generation

\section{INTRODUCTION}

For years, transportation engineers have studied the traffic and mobility patterns in cities. With the ever growing urban telecommunication networks, the amount of traffic information that can be collected, aggregated and redistributed is increasing. This facilitates the research on transportation systems and mobility patterns, but also introduces new problems related to the communication required among all the interconnected devices used to gather traffic data. Many researchers are working in a variety of areas that range from crowd-sourcing of information to safety applications. They are also making structural studies concerning infrastructure communications, traffic light systems, and more generally Intelligent Transportation Systems (ITS). In spite of the fact that both transportation engineers and the vehicular networking community are working on similar problems, the requirements and the scale of the tools that are used is different. The transportation community is mainly focused on traffic optimization and urban planning and is rarely interested in the behaviour of the single vehicle.
On the other hand, the networking community is concerned about the position and behaviour of the single vehicle and how this impacts on the communications and the related applications.

In order to study mobility patterns, traffic congestion or new communication protocols, we need a vehicular traffic simulator and an appropriate scenario to evaluate new proposals in a realistic environment. There are different kinds of traffic simulators that differ in the typology of the simulation that can be run (e.g. macro-, meso- and microscopic simulators). Macroscopic traffic simulators focus on the traffic flows, but do not take into consideration the single vehicle in the flow. For the vehicular networking community, the behaviour of a single vehicle is usually of interest and needs to be modelled carefully, thus microscopic simulators are generally preferred. Once the simulator is chosen, the common problem is that there are no properly-working and freely-available scenarios for the community.

Due to the lack of usable scenarios, the usual approach is to build a simple scenario that fulfills the purpose of the application. This approach results in several problems that are well known to the community, the most prominent being the lack of repeatable experiments allowing the comparison of different solutions or approaches that solve the same problem. Another problem that may be encountered is the specificity of the scenario and the consequent lack of generalization or realism.

In order to focus on Vehicle-to-X problems and solutions, the community needs a scenario that fulfills the following requirements: (1) It has to be able to support different kinds of traffic demand such as congested or free-flow patterns. (2) It should support different scenario dimensions. (3) It has to include different road categories (e.g. residential, arterial and highway). (4) It should allow multi-modal traffic evaluations (e.g. vehicles, public transport and pedestrian). (5) It should describe a realistic traffic scenario (i.e. avoid gridlocks and unrealistic mobility patterns) over one day in order to include the traffic mobility during rush hour (high density), during the day (moderate density) and during the night (low density). Our goal is to create a mobility scenario able to meet all the above mentioned requirements, and to make it freely available to the community in order to have a common reference for the 
different experiments.

We decided to use the road network of a real city as basis for our scenario in order to reproduce real traffic demand and mobility patterns. We chose the City of Luxembourg because its topology is comparable to that of many of European cities and because of available traffic statistics, which can be used to calibrate the traffic demand. Another advantage is that its size is reasonable in terms of complexity for a microscopic simulator.

The rest of the paper is organized as follows. Section II reviews the different traffic scenarios available to the vehicular networking community and explains their limitations. Section III explains in details how the LuST Scenario has been built covering aspects such as the road topology, the population and the traffic demand, and the different kind of vehicles involved. Section IV presents an overview of the possible use cases for the scenario. The paper concludes in Section V with a summary of the benefits and capabilities of the LusT Scenario and the future work.

\section{RELATED WORK}

Over the last few years, a great amount of work has been done by the vehicular networking community. New traffic and network simulation tools have been presented, and the problem of developing a reliable and usable mobility scenario has been encountered multiple times.

Among the vehicular traffic simulators we can find proprietary tools such as Visum [1] and VisSim [2], which are mainly used to study traffic flow optimizations and traffic demands. Multi-agent traffic simulators such as MATSim [3], are able to simulate the single vehicles, but they are more focused on the routing choices of the agents compared to the physics behind the behaviour of the vehicles. Another vehicular simulator is SUMO (Simulator of Urban MObility) [4], a microscopic traffic simulator that supports on-line interaction and close loop feedback through the TraCI [5] interface. It is able to simulate multi-modal traffic, traffic lights, inductive loops and other detectors.

Concerning the different scenarios available to the vehicular networking community, SUMO provides the TAPASCologne [6] scenario package, which includes road networks imported from OpenStreetMap (OSM) [7] and the traffic demand for the period between 6:00 and 8:00 in the morning. Unfortunately this scenario is difficult to use and requires additional work to improve the network quality, and to verify how routes are mapped onto both the network and the traffic demand.

In 2014, during the SUMO User Conference, a realistic traffic scenario from the city of Bologna [8] was released to the community. The scenario, built in the iTETRIS [9] framework, gives a very good starting point for the community, but it presents some limitations. The traffic demand is only defined over one hour, and the size of the scenario, which is relatively small, provides only one neighbourhood, lacking in generality.

Another possibility is the Sioux Falls test scenario [10]. This is a small scale, multi-modal test-bed with realistic traffic demand, integrates all the major features of MATSim such as points of interest, secondary location choice, and a population with heterogeneous socio-demographic parameters. In this case the scenario covers only the main streets. In addition, MATSim would not be easy to couple with a wireless networks simulator. The simulator implements a close loop feedback in order to optimize the mobility, but it does not provide an external interface to interact with it.

In 2015, ITS Austria West [11] presented a real life traffic monitoring system that uses a mesoscopic version of SUMO (this version of the simulator is not freely available). This project monitors a road network with 248,749 nodes and 323,282 road segments with a total length of $27,437 \mathrm{~km}$. They have five different traffic information sources providing floating car data and a traffic demand model for the simulation of 1.2 million routes and 1.6 million vehicles.

The ITS Austria West scenario is a very good example of the effort required to build and maintain a traffic scenario. There exist many other scenarios that are well designed, but these are not freely available to the community as they often rely on proprietary information.

With LuST Scenario we wanted to build a tool for the scientific community. In order to do so, we used only freely and publicly available datasets and software.

\section{LUST SCENARIO}

\section{Topology}

In order to create a realistic scenario we decided to start from a real mid-sized European city. The topology of many European cities consists of a central downtown area, surrounded by all its different neighbourhoods, which are linked by arterial roads [12]. Another important characteristic is the presence of a highway on the outskirts that surrounds the city. The size of the metropolitan area is another very important property: the scenario must be big enough to show the standard congestion patterns visible in modern cities, but it must be adequately small to permit simulations in a reasonable amount of time. The City of Luxembourg meets those requirements.

After choosing the city, we used OpenStreetMap (OSM) to extract its road topology. An OSM file contains all the necessary information about the environment and its accuracy has been detailed in [13]. We used JOSM [7] to extract and manually select and change points of interest and road segments. In this phase we retrieved information about roads (of any kind), traffic lights, locations and names of bus stops; we also saved additional information about schools (i.e. location and kind) to be used in the activity generation process, and the geometry of the buildings in order to create the polygons required for the obstacles, important component for wireless networks simulations.

\section{Mobility simulator}

The choice of the mobility simulator depends on the kind of studies that have to be done. In case of vehicular communications studies [14], the vehicular mobility has to be coupled with a network simulator, therefore a micromobilty simulator such as SUMO is appropriate for our needs. 


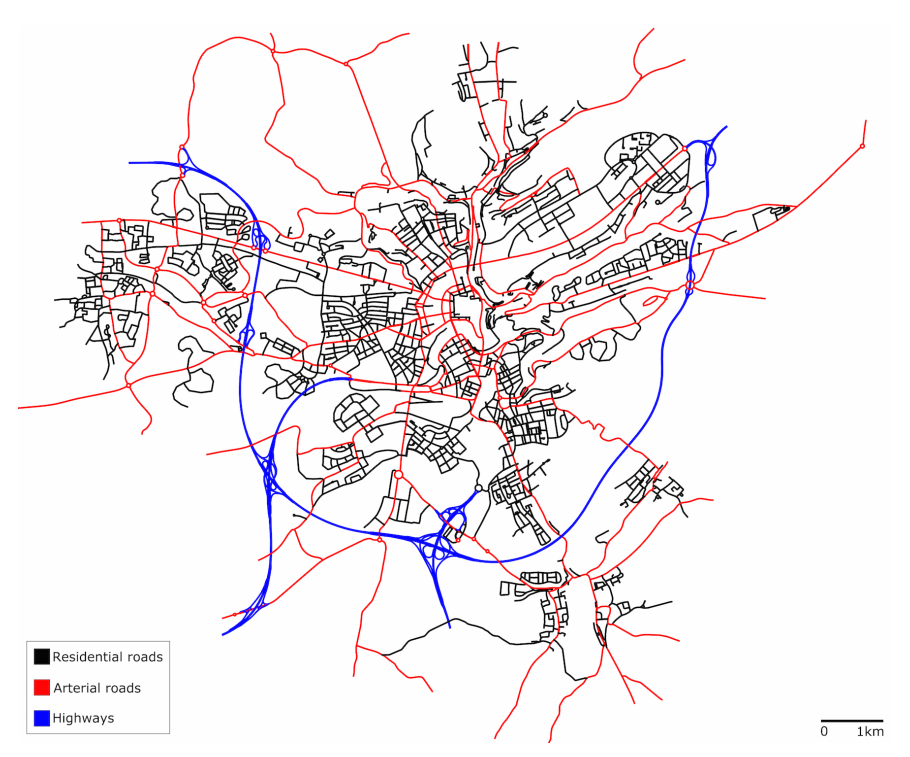

Fig. 1. LuST Scenario Topology.

As the aim of this scenario is to have a working SUMO simulation, all the intersections were checked manually for correctness. Using an iterative process with JOSM, netconvert [15] and SUMO, we ensure that no intersection represented an unrealistic bottleneck for the traffic flows. This iterative process was necessary in order to construct a road network with the proper intersection geometry and segment shape required by SUMO. These changes resulted in some minor differences in the topology (i.e. the angle between two streets may be different and that would slightly change the shape of the roads) that had to be taken into consideration while generating bus stops, bus routes and the polygons for the buildings.

To provide more flexibility, we decided not to impose any vehicle restrictions on any edge or lane (i.e. we removed the lanes reserved for specific type vehicles). In order to maintain the traffic patterns close to reality, we modified the number of lanes in some segments of the roads. We tried to standardise the roads in order to obtain a scenario that could easily be modified or extended.

Figure 1 shows the topology of the LuST Scenario, with streets coloured by type. The highway is depicted in blue, the main arterial roads in red and the residential roads in black. The static information contained in the roads network topology file is summarised in Tables I and II. The scenario covers an area of almost $156 \mathrm{~km}^{2}$ with a total of $931 \mathrm{~km}$ of roads of different types. In the SUMO network file, an edge is defined as a segment between two nodes, it may have a shape, and it is divided in one or more lanes.

\section{Demographics}

In order to achieve realistic traffic patterns we used the data published by the government, which is available on the Internet site of the Luxembourg National Institute of Statistics and Economic studies (STATEC) [16] (e.g. population, age
TABLE I

TOPOLOGY INFORMATION.

\begin{tabular}{|l|r|}
\hline Area & $155.95 \mathrm{~km}^{2}$ \\
\hline Total nodes & 2,372 \\
\hline Total edges & 5,969 \\
\hline Total length edges & $931.12 \mathrm{~km}$ \\
\hline Total length lanes & $1,571.4 \mathrm{~km}$ \\
\hline Edges with 1 lane & 3,944 \\
\hline Edges with 2 lanes & 1,188 \\
\hline Edges with 3 lanes & 764 \\
\hline Edges with 4 lanes & 78 \\
\hline
\end{tabular}

TABLE II

INTERSECTIONS INFORMATION.

\begin{tabular}{|l|r|}
\hline Roundabouts & 39 \\
\hline Total junctions & 4,341 \\
\hline Traffic lights & 203 \\
\hline Unregulated & 16 \\
\hline Priority & 1,914 \\
\hline Internal & 1,969 \\
\hline Dead end & 239 \\
\hline
\end{tabular}

distribution) to generate the activity demand for the ACTIVITYGEN [17] tool. The configuration files required by the tool must contain information concerning the topology of the city, demographic information about the population, schools, workplaces and residential areas. All of these are retrieved from OSM and STATEC.

\section{Mobility}

The traffic model is based on a mobility study that describes traffic characteristics over recent years [18]. We decided to tune the traffic demand between 200,000 and 300,000 vehicles per day, depending on which traffic demand we want to simulate (details are in Section Traffic Demand). The public transport database was used to retrieve the information about bus routes [19]. A total of 563 bus stops were added to the scenario. As shown in Table III, we added 38 bus routes inside the city for a total of 2,336 buses over the 24 hours period. The location of the bus stops in the LuST Scenario is not the same as the one in the OSM file (as mentioned earlier), however we tried to keep them as close as possible. For this reason, we had to rebuild the bus routes to match the new bus stop locations. Figure 2, depicts the recomputed bus routes (in red). Figure 3 shows an intersection located in the city centre; the yellow squares are the inductive loops positioned $5 \mathrm{~m}$ [20] from the intersection and the green boxes annotated with an $\mathrm{H}$ are the bus stops. With an additional configuration file it is possible to define location and sampling behaviour of the inductive loops. We positioned them at every intersection with a traffic light, on the highway, and on the on and off ramps (see Table IV). We fixed the location of each inductive loop close to the intersection to allow dynamic adjustments of the traffic light system using the information provided by the detectors as a feasible extension of the simulation. In case of the inductive loops situated on the highway, one of the possible usage is the monitoring of traffic flows on the peripheral roads. 


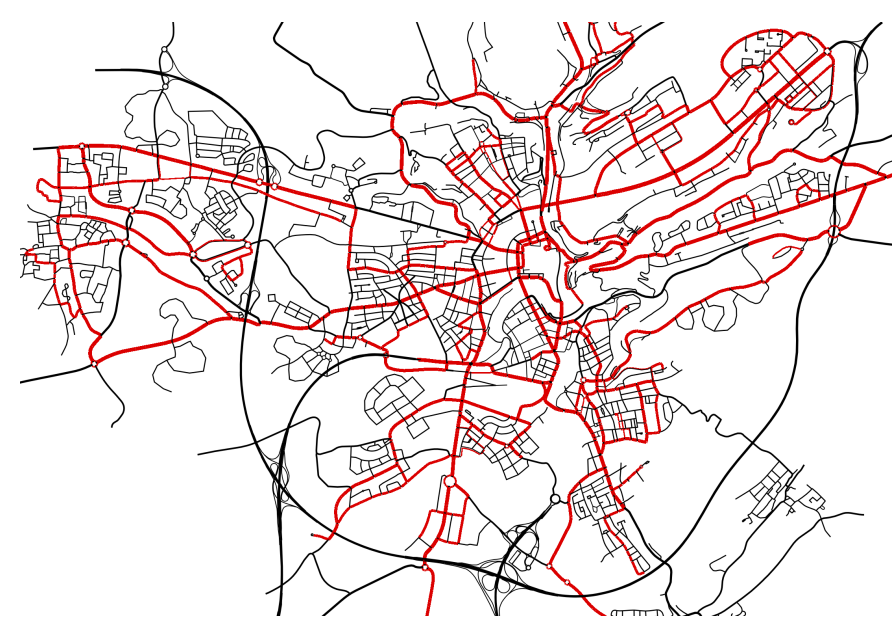

Fig. 2. Bus coverage in the LuST Scenario.

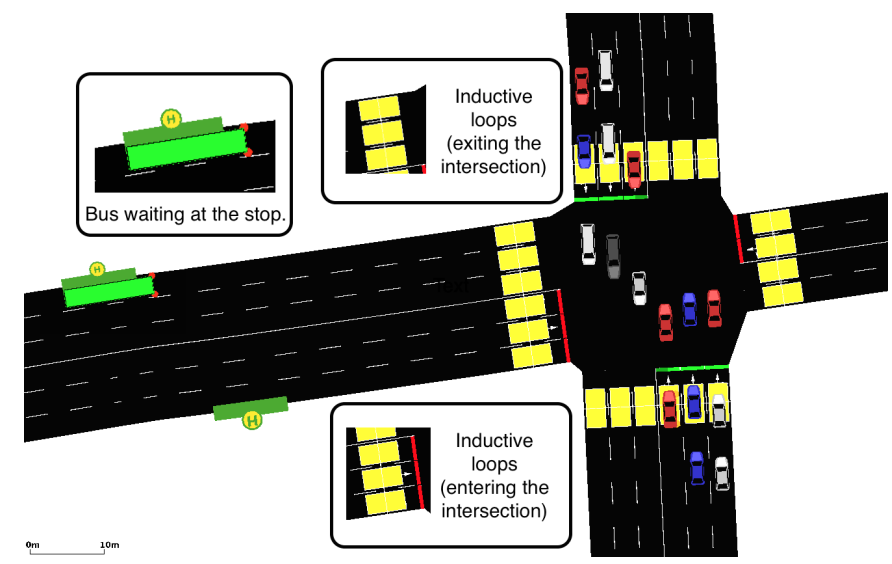

Fig. 3. Intersection with bus and inductive loops.

TABLE III

BUS INFORMATION

\begin{tabular}{|l|r|}
\hline Number of lines & 38 \\
\hline Bus stops & 563 \\
\hline Buses per day & 2,336 \\
\hline
\end{tabular}

TABLE IV

INDUCTIVE LOOP INFORMATION.

\begin{tabular}{|l|r|}
\hline Total number & 3,161 \\
\hline Highways & 94 \\
\hline Highway ramps & 225 \\
\hline Intersections & 2,842 \\
\hline
\end{tabular}

\section{Traffic demand}

The ACTIVITYGEN tool utilises the definition of a road network and the description of the population in order to generate a traffic demand for a scenario. It uses an activitybased traffic model that relies on a multi-modal trip planner including buses, cars, bicycles and pedestrians to derive the daily activities such as work, school, and free time. We separated the routes provided by the tools between vehicles and buses and optimised them using the SUMO duarouter
TABLE V

SUMO SIMULATION SHORT REPORT.

\begin{tabular}{|l|r|r|r|r|}
\hline & \multicolumn{2}{|c|}{ Comple Traffic } & \multicolumn{2}{c|}{ Local \& Buses Traffic } \\
\hline & Total & Percentage & Total & Percentage \\
\hline Inserted vehicles & 295,979 & & 218,938 & \\
\hline Teleports & 479 & 0.219 & 183 & 0.084 \\
\hline Collisions & 10 & 0.005 & 8 & 0.004 \\
\hline Jam & 142 & 0.065 & 55 & 0.025 \\
\hline Yield & 98 & 0.045 & 37 & 0.017 \\
\hline Wrong lane & 229 & 0.105 & 83 & 0.038 \\
\hline Emergency stops & 19 & 0.009 & 24 & 0.011 \\
\hline
\end{tabular}

tool. The complete traffic demand is composed of buses, and both transit and local traffic. A local vehicle has either a origin, or a destination, or both inside the city. A transit vehicle has both origin and destination outside the city and is mainly routed through the highway ring around the city. Depending on the purpose of the simulation, it is possible to leave out the transit traffic demand, keeping a realistic mobility for the urban area and improving the simulation speed.

The short report provided by SUMO at the end of the simulation for both the complete mobility and the local plus buses one is shown in Table V. In the percentage column we see that all the issues (e.g. teleports and emergency stops) that may be experienced by a vehicle during the simulation are lower than $0.2 \%$, indicating that the scenario is running smoothly without bottlenecks and gridlocks. The different traffic demands over the entire day are depicted in Figure 4. In red we find the overall traffic demand. The local and transit demands are depicted in blue and green respectively. We can clearly see the morning and evening rush hour peaks at 08:00 and 18:30 respectively and the off-peak period around lunch time. We distinguish between running vehicles $(\mathrm{R})$ and vehicles that are waiting to be inserted in the simulation (W). Figure 5 shows the distribution of the average speed. The two bells represent the urban traffic in the city and the extra-urban one on the highway. In order to provide a smooth mobility scenario, we use the dynamic routing mechanism provided by SUMO. We decided to allow the $70 \%$ of the vehicles in the simulation to change their route once every five minutes in order to react to being blocked in a traffic congestion. The motivation concerning the specific value are presented in Section IV. Table VI shows that the real number of vehicles that requires to be rerouted is reasonable. The $50 \%$ of the vehicles have never changed the route, 30\% have changed only once, $15 \%$ twice and only $5 \%$ of the vehicles changed route more than two times. This table proves that enabling the routing for $70 \%$ of vehicles does not create an unrealistic behaviours.

\section{Traffic patterns plausibility}

In order to show that the LuST Scenario behaves in a realistic way, we decided to compare it with the Typical Traffic option in Google Maps [21]. Figure 6a shows a snapshot of the simulation at 8:00 o'clock in the morning, and Figure $6 \mathrm{~b}$ shows the typical traffic on a Monday morning at the same time, provided by Google Maps. In this context, the relative 


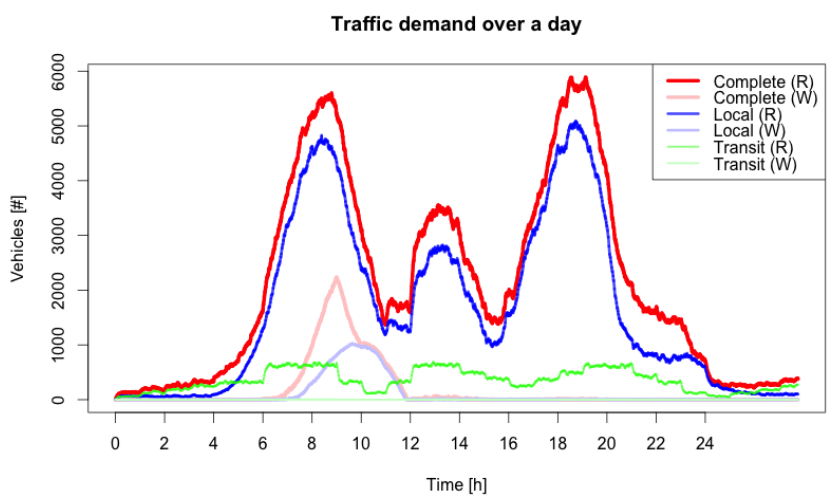

Fig. 4. Traffic Demand over a day. (R) represents the running vehicles and (W) the waiting ones at each given time.

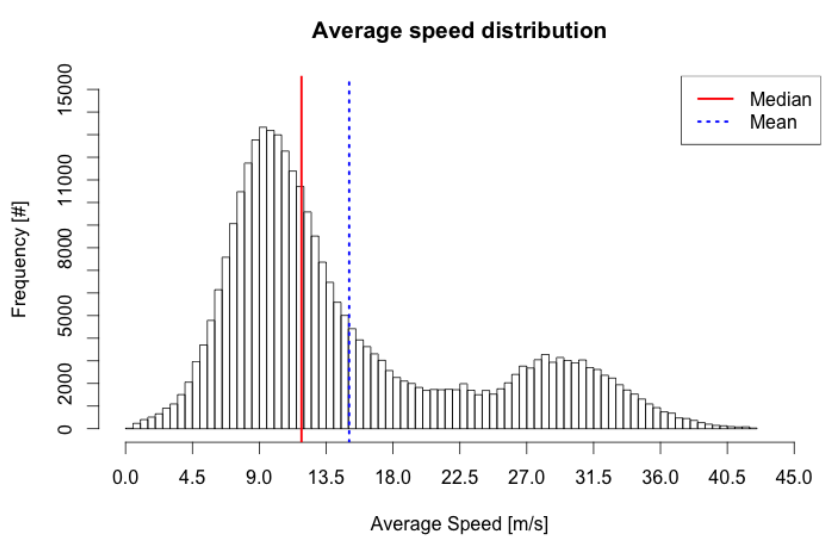

Fig. 5. Distribution of the average speed of the trips.

TABLE VI

Distribution OF THE NUMBER OF ROUTE CHANGES.

\begin{tabular}{|c|r|r|}
\hline Number of changes & Absolute & Percentage \\
\hline 0 & 147,224 & 49.80 \\
\hline 1 & 80,802 & 27.33 \\
\hline 2 & 43,675 & 14.77 \\
\hline 3 & 8,558 & 2.89 \\
\hline 4 & 2,528 & 0.85 \\
\hline $5+$ & 7,911 & 2.67 \\
\hline
\end{tabular}

speed is computed considering the speed limit on a segment. The colors, from green to red, represent the relative speed from normal to very congested. The traffic patterns are consistent over the 24 hours of simulation compared to the Google Maps information, allowing us to assume that the mobility provided by the LuST Scenario is realistic.

\section{Buildings}

In order to use the scenario with a network simulator such as NS3 [22] or OMNet++ [23] it is necessary to have information regarding the shape and position of the buildings. The information is extracted from OSM and refined with JOSM to match the modified network topology. In OSM the streets are defined

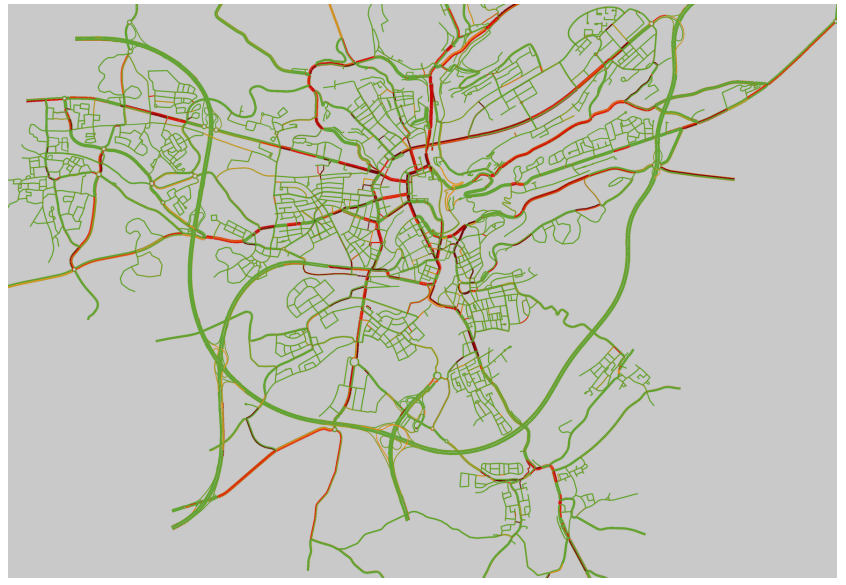

(a) Morning rush hour in LuST Scenario (8:00).

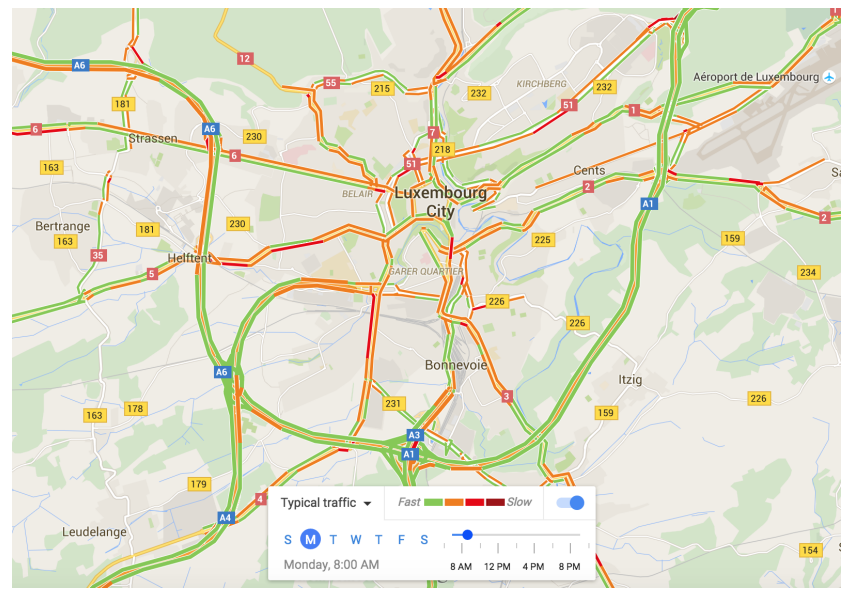

(b) Morning rush hour in Google Traffic (8:00).

Fig. 6. Relative speed (lanewise) during morning rush hour.

TABLE VII

POLYGONS INFORMATION IN THE LUST SCENARIO.

\begin{tabular}{|l|r|}
\hline Total & 14,171 \\
\hline Buildings & 13,553 \\
\hline Parking lots & 618 \\
\hline
\end{tabular}

as dots (intersections) and segments (roads) with a length but without the lane size. Once the street is converted for SUMO, the actual dimension of the lanes plays a crucial roles to avoid having buildings in the middle of the roads. Importing the geometry of the buildings directly from OSM would create an overlapping between the lanes and the edges of the polygons. Table VII summarize the polygons imported in the scenario. We decided to have only two different categories of polygons for the moment. The parking lots are important for the studies concerning the mobility, for example to evaluate multi-modal strategies. The other polygon type represents the buildings (including apartments, houses and construction sites), which are fundamental to evaluate communication protocols and obtain realistic results. In Figure 7 shows the location of the buildings (in red) and the parking lots (in grey) in a business district of the LuST Scenario. 


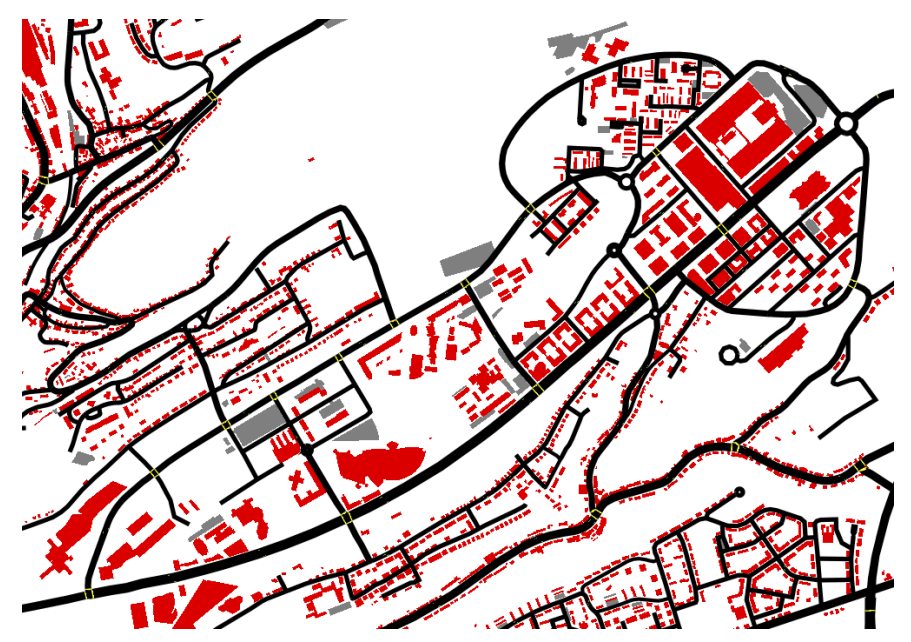

Fig. 7. A detail of the buildings and parking lots information in the LuST Scenario - business district.

\section{UsE CASES}

The LuST Scenario is a framework that provides realistic mobility patterns for a mid-size city. The mobility traces provided can be used as an input for other types of simulators such as NS3 or OMNet++ in order to evaluate network protocols. We will now show how the LuST scenario can be used in association with Veins [24] to obtain a closedloop feedback between SUMO and OMNet++. Using LuST in combination with those tools allows the study of both, the performance of Vehicular-to-X protocols and the related applications. Further, the scenario allows to evaluate different multi-modal strategies (e.g. for commuters). Using Veins, it is possible to confine the computation of the connectivity network to a restricted area, enabling the simulation of a smaller traffic scenario that only uses a subset of the available road network, allowing testing of protocols and applications on different scales without changing the mobility patterns. In our urban area there are 203 intersections managed by a traffic light system. These intersections can be used to test different optimisation strategies (e.g. green waves) or emergency protocols to allow firefighters, ambulances or the police to be prioritised.

In the next paragraph we explore in more detail two possible use cases of the LuST Scenario. In the first, we vary the percentage of vehicles allowed to change their route in case of congestion, and in the second we use Veins and OMNet++ to compute the average number of first-hop neighbours that connected vehicles encounter in a smaller area of the city.

\section{Routing}

For the default scenario, $70 \%$ of the vehicles are equipped with a routing device, allowing to change route if a congestion is encountered. We motivate this parameter by the fact that most of the local commuters are familiar with the road network and know at least one alternative path. Further, more and more cars are equipped with real time navigation services allowing a better usage of the available road capacity.
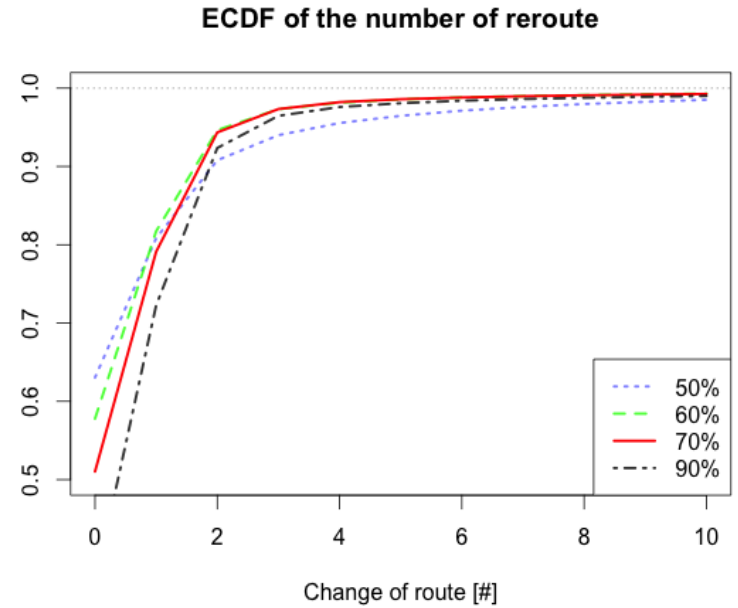

Fig. 8. Empirical Cumulative Distribution Function of the number of route changes in the different scenarios.

In SUMO, each vehicle can be equipped with different kinds of devices in order to interact with their environment. Using the on-board routing system, it is possible to change the percentage of cars that react to their surroundings, obtaining scenarios with different levels of congestion to test different routing strategies. We decided to vary the percentage of vehicles equipped with the routing device from $50 \%$ to $90 \%$ and evaluate the impact on the number of route changes for each vehicle and the impact on the experienced waiting time. Figure 8 present the Empirical Cumulative Distribution Function (ECDF) for the number of route changes in the experiments, we see that lowering the percentage of vehicles that are allowed to react to traffic congestion increases the number of route changes for the ones that are equipped with a routing system. Nevertheless, even with only $50 \%$ of the vehicles equipped with the routing device, $95 \%$ of the vehicles in the simulation change their route less than 4 times.

The waiting time experienced by the vehicles is another interesting aspect impacted by the percentage of vehicles equipped with a routing system. The waiting time is defined as the number of seconds in which the vehicle speed is lower than $0.1 \mathrm{~m} / \mathrm{s}$ during the simulation. Given its definition, the waiting time is a fair measurement of traffic congestion. Figure 9 present its ECDF. Here the difference between the experiments is more visible. In case of $50 \%$ of vehicles equipped with the device, we see that more than $15 \%$ of the vehicle spend more than 30 minutes blocked in traffic, which, for this scenario, represents 45.000 vehicles. In case of $70 \%$ of vehicles equipped with the device (default value) only $2 \%$ or $3 \%$ of the vehicles will experience a waiting time longer than 30 minutes.

It is important to consider that the shape of the traffic demand will change by varying the percentage of rerouted vehicles. The three-bell shaped traffic demand that represent the three rush hours (morning, noon and evening) is always 


\section{ECDF of the waiting time}

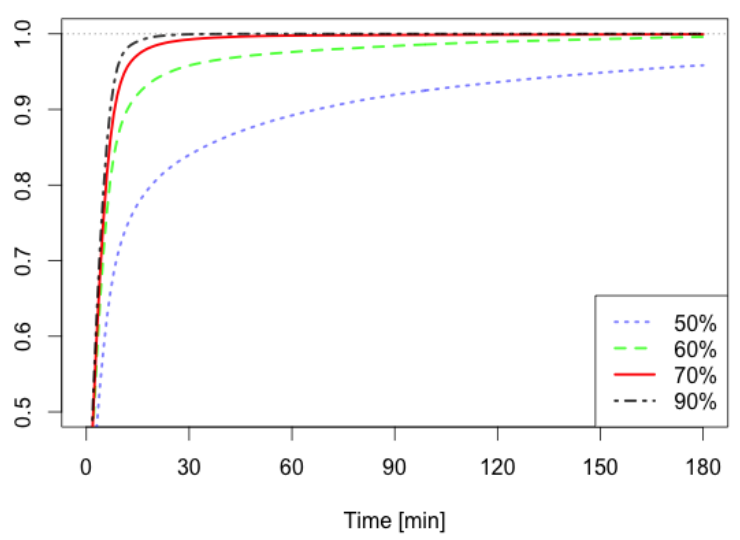

Fig. 9. Empirical Cumulative Distribution Function of the waiting time in the different scenarios.

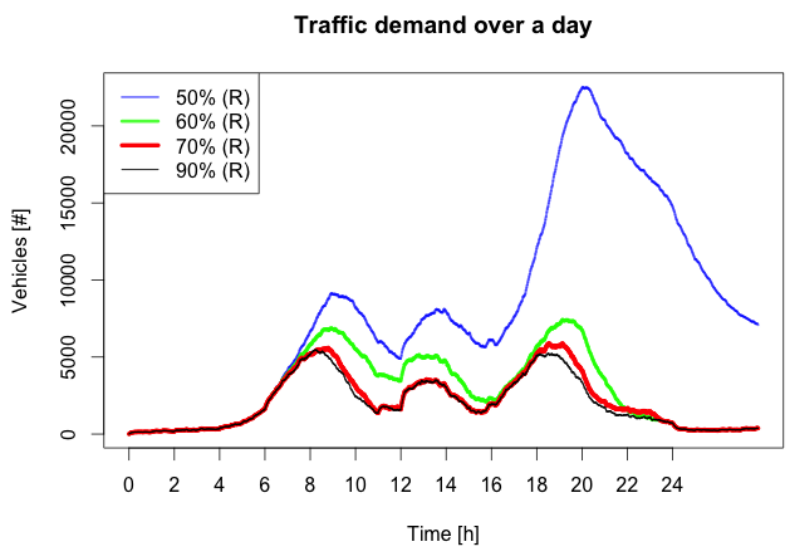

Fig. 10. Comparison of the different traffic demands in the different scenarios.

present, but with $50 \%$ or less vehicles allowed to change route, the traffic congestion will significantly increase and the mobility scenario will become unrealistic. In fact, as shown in Figure 10, all the rush hour peaks will be delayed due to a congested network.

The routing used by the on-board devices provided by SUMO, represents the First Wardrop Principle of equilibrium [25], the selfish user approach. In this case, the vehicles have complete information concerning the overall traffic situation in order to take the best decision. A more detailed study concerning the impact of this kind of behaviour can be found in [26].

\section{Clustering}

With this use case we show how to reduce the size of the experiment to a smaller area and use a wireless network simulator in order to have an estimation of the number of vehicles (cluster) encountered during a trip.
TABLE VIII

STATISTICS OF THE BUSINESS DISTRICT SCENARIO

\begin{tabular}{|l|r|r|r|}
\hline & Median & Mean & St. Deviation \\
\hline Trip length [m] & $1,226.94$ & $1,517.49$ & $1,287.68$ \\
\hline Speed [m/s] & 13.03 & 13.84 & 5.27 \\
\hline \multicolumn{4}{|c|}{ With Buildings } \\
\hline Veh. enc per sec & 6.59 & 8.09 & 6.38 \\
\hline \multicolumn{4}{|c|}{ Without Buildings } \\
\hline Veh. enc per sec & 14.62 & 16.18 & 9.52 \\
\hline
\end{tabular}

We used OMNet++ with Veins to simulate a IEEE 802.11p [27] on-board unit that broadcast beacons every second to compute the number of vehicles in range. In this case all the vehicles are equipped with the $802.11 \mathrm{p}$ device.

Figure 7 shows the business district of Luxembourg, an area of 3.6 by 3.3 kilometres in which at north-east there is one exit/entrance to the highway and at south-west there is a bridge to the city centre. The resizing of the simulation scenario to this area can be done both in SUMO and in OMNet++. Reshaping the scenario in SUMO will imply a change in the mobility pattern given that all the vehicles with an origin outside the area will not be simulated. Restricting the simulated area in OMNet++ will keep the realistic mobility pattern but will reduce the channel computation to the restricted area.

Table VIII presents the statistics of this experiment. The resized simulation is composed of 43,443 vehicles over a period of 9 hours, from $9 \mathrm{~h} 00$ until $18 \mathrm{~h} 00$. In the table, the row "Vehicles encountered per second" represent the median, mean and variation of the cluster size.

This experiment has been conducted in order to have an estimation of the clustering of this scenario. In the TAPAS Cologne paper [6] the authors provided an extensive evaluation of the clustering of the vehicles for their scenario compared to two mobility traces for the cities of Turin and Zurich. We were not able to obtain the latter so we rely on the findings referenced in the TAPAS Cologne paper. The aim is to prove that the scenario is realistic and that this metric is crucial in order to have reliable result in case of the evaluation of a network protocol. Their argument is about the fact that in the other mobility traces, given the higher degree of connected nodes, the network would result more stable, mining the reliability of the results obtained for the evaluation of a network protocol, and the same architecture with the other traces could lead to over-optimistic results. We completely agree with this conjecture, and we would like to add that the presence of the building in the LuST Scenario brings a higher level of realism as the signal propagation is considered. In order to be comparable with the TAPAS Cologne scenario, given that the presence of buildings is never mentioned, we run the same experiment with and without polygons for the channel simulation. As expected, in case of simulation without buildings, the average size of the clusters is larger compared to the one with the polygons (from approximatively 16 to approximatively 8 nodes), and this has an impact on the reliability of the evaluation [28]. 


\section{CONCLUSION AND FUtURE WORK}

In this paper we have introduced a traffic scenario built for the vehicular networking research community. The Luxembourg SUMO Traffic (LuST) Scenario meets all the common requirements needed for a common basis for the evaluation of network protocols and related applications. To build this scenario we started from a real mid-size city, with a typical European road topology and mobility patterns. The LuST scenario covers an area of $156 \mathrm{~km}^{2}$ and $932 \mathrm{~km}$ of roads. There are 38 different bus routes with 563 bus stops. All intersections with traffic lights and all highway ramps are equipped with inductive loops. We generate the traffic demand using real information provided by various data sources. We have discussed several use cases for the LuST Scenario. Among them are the evaluation and testing of network protocols, and applications for Intelligent Transportation Systems (ITS).

As new features are provided by the SUMO simulator, the scenario can be enriched with other transportation modes (e.g. pedestrian, bicycle). At the moment the traffic light system uses a static scheduler; among the additional functionality we want to provide, is a dynamic version of the traffic light system.

The preliminary version of the LuST Scenario has been presented at the SUMO User Conference 2015. Since then, the traffic demand and the polygons have been improved. The scenario is freely available under the MIT license to the whole community and it is hosted on GitHub (https://github.com/lcodeca/LuSTScenario).

\section{ACKNOWLEDGMENTS}

This AFR project with the project number 5761149 is funded by the Fond Nationale de la Recherche (FNR), Luxembourg.

\section{REFERENCES}

[1] "Visum official Internet site." http://vision-traffic.ptvgroup.com/enuk/products/ptv-visum/, August 2015.

[2] M. Fellendorf, "VISSIM: A microscopic simulation tool to evaluate actuated signal control including bus priority," in 64th Institute of Transportation Engineers Annual Meeting, 1994, pp. 1-9.

[3] M. Balmer, K. Meister, M. Rieser, K. Nagel, and K. W. Axhausen, Agent-based simulation of travel demand: Structure and computational performance of MATSim-T. ETH, Eidgenössische Technische Hochschule Zürich, IVT Institut für Verkehrsplanung und Transportsysteme, 2008.

[4] D. Krajzewicz, J. Erdmann, M. Behrisch, and L. Bieker, "Recent development and applications of SUMO-simulation of urban mobility," International Journal On Advances in Systems and Measurements, vol. 5, no. 3 and 4, pp. 128-138, 2012.

[5] A. Wegener, M. Piórkowski, M. Raya, H. Hellbrück, S. Fischer, and J.-P. Hubaux, "TraCI: an interface for coupling road traffic and network simulators," in Proceedings of the 11th communications and networking simulation symposium. ACM, 2008, pp. 155-163.

[6] S. Uppoor and M. Fiore, "Large-scale urban vehicular mobility for networking research," in Vehicular Networking Conference (VNC), 2011 IEEE. IEEE, 2011, pp. 62-69.

[7] M. Haklay and P. Weber, "Openstreetmap: User-generated street maps," Pervasive Computing, IEEE, vol. 7, no. 4, pp. 12-18, 2008.

[8] L. Bieker, D. Krajzewicz, A. Morra, C. Michelacci, and F. Cartolano, "Traffic simulation for all: a real world traffic scenario from the city of Bologna," in SUMO2014 - Modeling Mobility with Open Data. Deutsches Zentrum fur Luft- und Raumfahrt e.V. Institut fur Verkehrssystemtechnik, 2014.
[9] D. Krajzewicz, R. J. Blokpoel, F. Cartolano, P. Cataldi, A. Gonzalez, O. Lazaro, J. Leguay, L. Lin, J. Maneros, and M. Rondinone, "iTETRIS - A System for the Evaluation of Cooperative Traffic Management Solutions," in Advanced Microsystems for Automotive Applications 2010. Springer, 2010, pp. 399-410.

[10] A. Chakirov, "Enriched Sioux Falls Scenario with Dynamic Demand," in MATSim User Meeting. Springer, June 2013, pp. 15-34.

[11] K.-H. Kastner and P. Pau, "Experiences with SUMO in a Real-Life Traffic Monitoring System," in SUMO2015 - Intermodal Simulation for Intermodal Transport. Deutsches Zentrum fur Luft- und Raumfahrt e.V. Institut fur Verkehrssystemtechnik, 2015.

[12] P. Crucitti, V. Latora, and S. Porta, "Centrality measures in spatial networks of urban streets," Physical Review E, vol. 73, no. 3, p. 036125, 2006.

[13] M. Haklay, "How good is volunteered geographical information? A comparative study of OpenStreetMap and Ordnance Survey datasets," Environment and planning. B, Planning \& design, vol. 37, no. 4, p. $682,2010$.

[14] F. J. Martinez, C. K. Toh, J.-C. Cano, C. T. Calafate, and P. Manzoni, "A survey and comparative study of simulators for vehicular ad hoc networks (VANETs)," Wireless Communications and Mobile Computing, vol. 11, no. 7, pp. 813-828, 2011.

[15] "NETCONVERT wiki page." http://sumo.dlr.de/wiki/NETCONVERT, August 2015.

[16] "Luxembourg National Institute of Statistics and Economic internet site." https://www.statistiques.public.lu, August 2015.

[17] “ACTIVITYGEN wiki page.” http://sumo.dlr.de/wiki/ACTIVITYGEN, August 2015.

[18] "LuxTram official Internet site." https://www.luxtram.lu, August 2015.

[19] "The Luxembourg Mobility Internet site." https://www.mobiliteit.lu, August 2015.

[20] P. Koonce, L. Rodegerdts, K. Lee, S. Quayle, S. Beaird, C. Braud, J. Bonneson, P. Tarnoff, and T. Urbanik, "Traffic signal timing manual," Tech. Rep., 2008.

[21] R. C. Fan, X. Yang, and J. D. Fay, "Using location data to determine traffic information," Jul. 15 2003, uS Patent 6,594,576.

[22] G. F. Riley and T. R. Henderson, "The ns-3 network simulator," in Modeling and Tools for Network Simulation. Springer, 2010, pp. 1534.

[23] A. Varga and R. Hornig, "An overview of the OMNeT++ simulation environment," in Proceedings of the 1st international conference on simulation tools and techniques for communications, networks and systems \& workshops. ICST (Institute for Computer Sciences, SocialInformatics and Telecommunications Engineering), 2008, p. 60.

[24] C. Sommer, R. German, and F. Dressler, "Bidirectionally coupled network and road traffic simulation for improved IVC analysis," Mobile Computing, IEEE Transactions on, vol. 10, no. 1, pp. 3-15, 2011.

[25] J. G. Wardrop, "ROAD PAPER. SOME THEORETICAL ASPECTS OF ROAD TRAFFIC RESEARCH." in ICE Proceedings: Engineering Divisions, vol. 1, no. 3. Thomas Telford, 1952, pp. 325-362.

[26] L. Codeca, R. Frank, and T. Engel, "Improving traffic in urban environments applying the Wardrop equilibrium," in Network Protocols (ICNP), 2013 21st IEEE International Conference on. IEEE, 2013, pp. 1-6.

[27] D. Jiang and L. Delgrossi, "IEEE 802.11 p: Towards an international standard for wireless access in vehicular environments," in Vehicular Technology Conference, 2008. VTC Spring 2008. IEEE. IEEE, 2008, pp. 2036-2040

[28] F. J. Martinez, C.-K. Toh, J.-C. Cano, C. T. Calafate, and P. Manzoni, "Realistic radio propagation models (RPMs) for VANET simulations," in Wireless Communications and Networking Conference, 2009. WCNC 2009. IEEE. IEEE, 2009, pp. 1-6. 